\title{
Reply to letter by Dr. G. Corso
}

\author{
Frederik Stuebs $^{1} \cdot$ Simone Heidemann ${ }^{2,3} \cdot$ Almuth Caliebe $^{2} \cdot$ Christoph Mundhenke $^{1} \cdot$ Norbert Arnold $^{1}$
}

Received: 16 January 2018 / Accepted: 18 January 2018 / Published online: 28 February 2018

c) Springer-Verlag GmbH Germany, part of Springer Nature 2018

We would like to thank Giovanni Corso et al. for their "Letter to the editor". In our study, we screened 97 individuals for $\mathrm{CDH} 1$ mutations. Screening revealed two missense variants in independent families. The first alteration, A592T, was classified as neutral. The other variant, S838G, was detected in an unaffected woman with a family history of BRCAIpositive breast- and ovarian cancer without gastric cancer. Our index case was tested negative for the familial BRCAl mutation. In the past, the variant S838G has been classified as potentially deleterious [1]. Searching the AlamutDatabase (Alamut Visual 2.10) revealed contradictory rating of the variant from likely benign to pathogenic, whereas the pathogenic classification probably also was judged by the paper of Risinger et al. [1]. In addition, in dbSNP, one will find this spectrum of rating (rs121964872). A request to Ambry Genetics for the reason why they judge the variant as likely benign revealed following answer: The serine at codon 838 is replaced by glycine, an amino acid with similar properties. This amino acid position is well conserved in available vertebrate species. This variant has been seen in several families whose clinical histories are not consistent with hereditary diffuse gastric cancer or other CDH1-related cancers (Ambry Internal data). In addition, this alteration is predicted to be benign and tolerated by PolyPhen and SIFT in silico analyses, respectively. Based on the majority of available evidence to date, this variant is unlikely to be pathogenic. Therefore, the cases of breast and ovarian cancer in the reported family are most likely caused by the BRCAI

Frederik Stuebs

frederik.stuebs@uk-erlangen.de

1 Department of Gynaecology and Obstetrics, University Hospital of Schleswig-Holstein, Campus Kiel, ChristianAlbrechts University Kiel, Kiel, Germany

2 Department of Human Genetics, University Hospital Schleswig-Holstein, Campus Kiel, Christian-Albrechts-Unive rsity, Kiel, Germany

3 Present Address: Institute of Tumor Genetics North, Kiel, Germany mutation. Unfortunately, we had no possibilities to test the segregation of this missense variant S838G in other family members. This is why, we cannot exclude that this $\mathrm{CDH} 1$ variant could have a modifying influence on breast cancer causing the lobular histology.

The clinical management option mentioned in the letter of Corso et al. should be regarded with caution if no clear deleterious alteration is found in the gene, because the clinical consequences for the patient not only psychologically but also physically are very harmful. Due to the present evidence regarding the missense variant $\mathrm{S} 838 \mathrm{G}$, we presently do not see any need for clinical intervention.

However, we agree that, based on the literature, there is a connection between $\mathrm{CDHI}$ alteration and the lobular type of breast cancer, and this may fulfill the criteria of an independent syndrome.

\section{Compliance with ethical standards}

Conflict of interest The author declare that there is no interest of conflict for all authors.

\section{Reference}

1. Risinger JI, Berchuck A, Kohler MF, Boyd J (1994) Mutations of the E-cadherin gene in human gynecologic cancers. Nat Genet 7:98-102 